\title{
Hedgehog signaling pathway in colorectal cancer: function, mechanism, and therapy
}

OncoTargets and Therapy

30 June 2017

Number of times this article has been viewed

\section{Chuanqing Wu \\ Xiaojie Zhu \\ Weizhen Liu \\ Tuo Ruan \\ Kaixiong Tao}

Department of Gastrointestinal Surgery, Union Hospital, Tongji

Medical College, Huazhong

University of Science and

Technology, Wuhan, China
Correspondence: Kaixiong Tao

Department of Gastrointestinal Surgery, Union Hospital, Tongji Medical College,

Huazhong University of Science and

Technology, Wuhan 430022, China

Tel +86278572 1619

Email taokaixiong19@163.com

\begin{abstract}
Colorectal cancer (CRC) is one of the most common gastrointestinal cancers worldwide. It is a complicated and often fatal cancer, and is related to a high disease-related mortality. Around $90 \%$ of mortalities are caused by the metastasis of CRC. Current treatment statistics shows a less than 5\% 5-year survival for patients with metastatic disease. The development and metastasis of CRC involve multiple factors and mechanisms. The Hedgehog (Hh) signaling plays an important role in embryogenesis and somatic development. Abnormal activation of the Hh pathway has been proven to be related to several types of human cancers. The role of Hh signaling in $\mathrm{CRC}$, however, remains controversial. In this review, we will go through previous literature on the Hh signaling and its functions in the formation, proliferation, and metastasis of CRC. We will also discuss the potential of targeting Hh signaling pathway in the treatment, prognosis, and prevention of CRC.
\end{abstract}

Keywords: colorectal cancer, Hedgehog signaling pathway, cancer therapy

\section{Introduction}

Colorectal cancer (CRC) is one of the most common malignancies worldwide, which is related to significant morbidity and mortality. Despite the advancements in diagnosis and treatment of this tumor type, identification of new prognostic biomarkers continues to be a challenge.

The Hedgehog (Hh) signaling pathway plays an essential role in the patterning, growth, and differentiation in various tissues, including the gastrointestinal tracts. ${ }^{1-5}$ In mammals, Hh signaling initiates with binding of one of the three ligands - Sonic Hedgehog (Shh), Indian Hedgehog (Ihh), and Desert Hedgehog (Dhh) - to the transmembrane receptor patched 1 (Ptc1), causing the release of the suppressed transmembrane protein Smoothened (Smo), which is a member of the seven transmembrane-receptor family, most closely related to the Frizzled family. ${ }^{2}$ The release of Smo subsequently activates the Gli transcription factors. ${ }^{3,6} \mathrm{Hh}$ signaling pathway has been implicated in the pathogenesis of various human cancers; however, the role of the Hh pathway in CRC remains controversial. ${ }^{6}$ There are two mechanisms of Hh activation: through $\mathrm{Hh}$ ligand-dependent activation or through ligand-independent activation, namely, by loss-of-function mutations in Ptc1 or gain-of-function mutations in the proto-oncogene $S m o .^{7}$ The Hh pathway is viewed as a cancer-related pathway and a potential therapeutic target.

In this review, we will go through previous literature on the Hh signaling pathway and its functions in the formation, proliferation, and metastasis of CRC. We will also discuss the potential roles of the $\mathrm{Hh}$ signaling pathway in the treatment, prognosis, and prevention of CRC. 


\section{Hh signaling pathway}

Since first discovered in Drosophila melanogaster, the Hh signaling pathway has received extensive attention. ${ }^{1-7}$ Massive research has confirmed its contributions in embryonic generation and postembryonic regulation of the development of various organs and tissues, including the patterning, growth, and differentiation in the gastrointestinal tracts. ${ }^{1-5}$ The Hh signaling pathway is involved in the continuous renewal of the intestinal epithelial cells in adults, which leads to the speculation that dysregulation of the Hh signaling pathway would cause pathological hyperplasia of intestinal epithelial cells and contribute to the generation and progression of malignancies. ${ }^{2,5,8}$

There are three mammalian Hh homologs - Shh, Ihh, and Dhh - in vertebral animals that participate in the patterning and development of various tissues and organs. ${ }^{2,8}$ These homologs are also functional in certain types of tissue regeneration and tumorigenesis. ${ }^{910}$ In the gastrointestinal tracts, both Shh and Ihh are expressed, while Dhh expression appears to be restricted to the nervous system and testes. ${ }^{10}$ The major components of the Hh pathway are localized to the cell membrane. ${ }^{11}$ The transduction response to Hh ligands is regulated and conveyed by two transmembrane proteins: Ptc and Smo, and by the downstream transcription factors of the Gli family (Gli1, Gli2, and Gli3). ${ }^{12}$

The Hh signaling pathway begins with the production and secretion of Hh ligands. ${ }^{13}$ The Hh ligands then bind to Ptc and subsequently activate the G protein-coupled receptor-like transmembrane protein Smo. ${ }^{14}$ When $\mathrm{Hh}$ is absent, Ptc inhibits Smo via an unknown mechanism. ${ }^{15}$ By binding to Hh, the Smo-repressing activity of Ptc is inhibited, thus freeing Smo to exhibit its signal activity intracellularly. Smo is located in primary cilia and signals intracellularly to mediate the three Gli zinc finger transcription factors. ${ }^{16,17}$ Gli proteins are the last molecules of the pathway and the key final output of $\mathrm{Hh}$. In vertebrates, there are three members in the Gli gene family: Gli1, Gli2, and Gli3. Gli1 is an $\mathrm{Hh}$ response gene product that functions only as a transcriptional activator and is involved in a positive feedback circle upon pathway activation. Gli2 and Gli3 possess opposite functions: Gli2 functions primarily as a transcriptional activator, while Gli3 serves as the primary transcriptional inhibitor. ${ }^{18}$ Smo is capable of activating two different intracellular signaling cascades: a non-canonical, ligand-independent pathway that modulates the cytoskeleton by modulating Rac1 and Rho1 GTPases and a canonical, ligand-dependent pathway through Gli2 activation. ${ }^{18}$ Smo-regulated canonical signaling pathway involves intracellular activation of Gli2 by limited proteolysis. Full-length Gli2 resides in the cytoplasm linked to a suppressor complex composed of Fused kinase (Fu), Suppressor of Fused ( $\mathrm{SuFu}$ ), and Costal2. Smo activation releases Gli2 from the suppressor complex and transfers it to the nucleus to bind to the gene promoters induced by Hh signaling. Gli2-mediated Hh signaling requires the participation of its receptor Ptc, Hedgehog interacting protein (Hhip), and the transcription factor Gli1. ${ }^{19}$ Thus, Gli1, Ptc, and Hhip are general transcriptional targets of canonical Hh signaling activity. ${ }^{20}$ In the absence of Ptc ligand, Smo is inactive, thereby inhibiting the transcription of Gli1 and the release of Gli2, and Gli3 is cleaved to generate repressor isoforms (Gli3Rs). When Smo is activated by Ptc, Gli2 is released from the cytoplasm complex; Gli3 repressor function is inhibited; and Gli1 is transcriptionally active, combined together, the final output is generally transcription of Gli1 and Gli2 target genes (Figure 1). ${ }^{16}$

The inappropriate activation of Hh pathway is frequently found in various tumors, including basal cell carcinoma, medulloblastoma, pancreatic cancer, lung cancer, breast cancer, and gastric cancer. ${ }^{12,18-21}$ Deregulation of the $\mathrm{Hh}$ pathway can occur in cancers either by mutations in key effectors of the canonical signaling pathway or by aberrant expression of $\mathrm{Hh}$ itself. ${ }^{22,23}$ Some studies have also revealed that CRC cells, which frequently express Hh ligands, are believed to exert paracrine effects on the stromal component of the tumor. ${ }^{24}$ However, the role of the Hh signaling pathway in CRC remains controversial. ${ }^{6}$ The results vary among studies: according to currently available data, while most studies showed a correlation between Hh and CRC (98 out of 101 studies), there are three researches that claimed that $\mathrm{Hh}$ is not, or at least not directly, related to CRC. ${ }^{25-27}$ Within the 98 studies, 92 studies confirmed upregulation effects and 5 revealed downregulation effects of the Hh pathway in CRC. ${ }^{435,40,42,47-49,53-113}$ Moreover, among the studies in favor of a Hh-CRC correlation, its exact function in the formation, proliferation, drug resistance, and metastasis of CRC is not uniform.

\section{Colorectal cancer}

$\mathrm{CRC}$ is one of the most common gastrointestinal cancers in the world. It is a complicated and often fatal cancer. ${ }^{28,29}$ Despite the overall therapeutic improvements, there is still a high disease-related mortality (about 33\%). ${ }^{30}$ Approximately $90 \%$ of the mortality was caused by the metastasis of CRC.${ }^{31}$ Current clinical statistical data show less than 5\% 5-year survival for metastatic CRC.32 The most common type of CRC is sporadic CRC, which makes up to nearly $80 \%-85 \%$ of all CRC cases. ${ }^{33}$ 

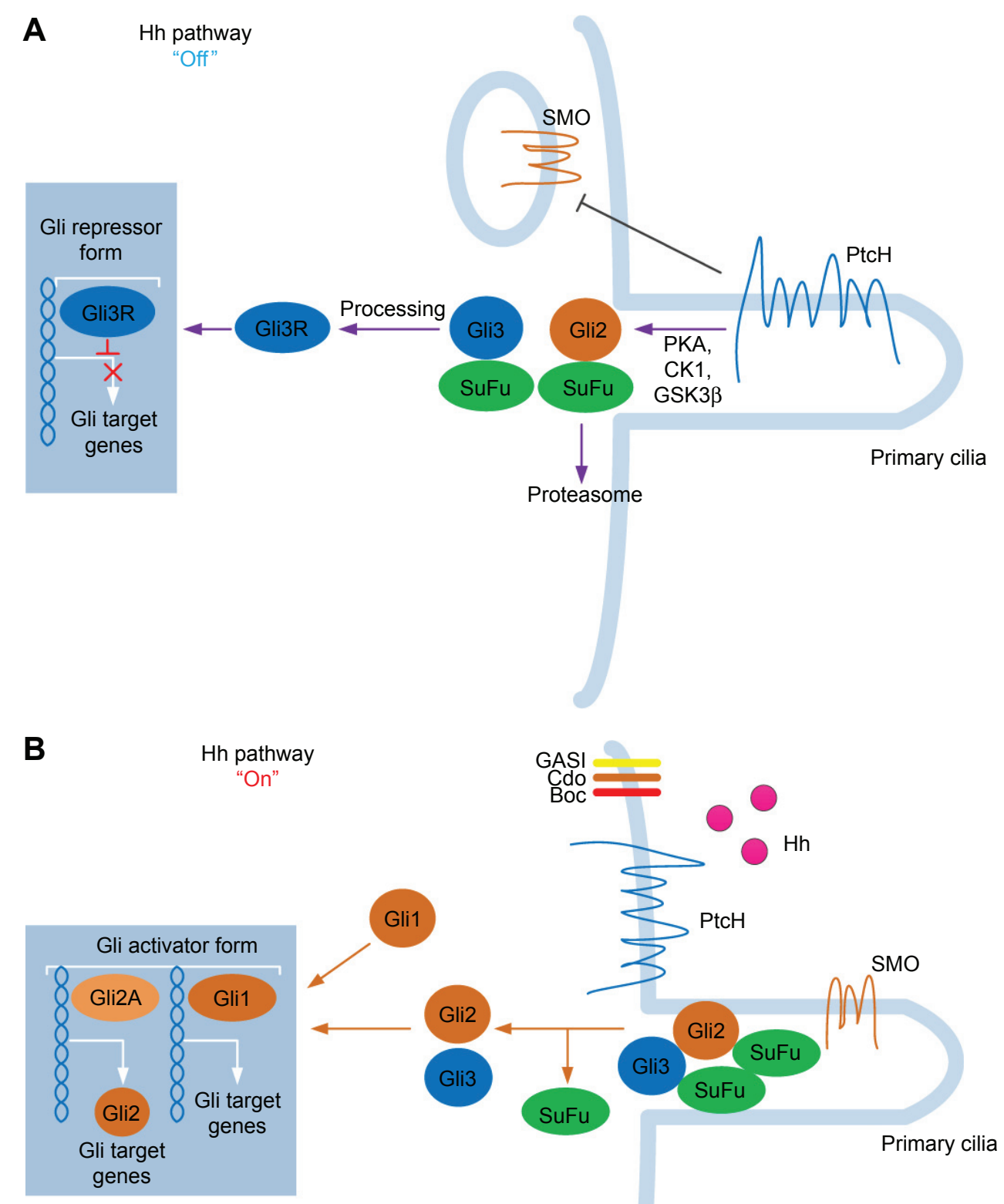

Figure I The sketch of Hedgehog $(\mathrm{Hh})$ signaling pathway. The Hh signaling pathway contains three Hh homologs: Sonic Hh, Indian Hh, and Desert Hh. (A) When the ligand is absent ("Off" state), the patched (Ptc) receptor inhibits the downstream protein Smoothened (SMO). Henceforth, glioma-associated oncogene homolog (Gli) proteins are sequestered by Suppressor of Fused (SuFu). The Hh pathway is, generally, inhibited at "Off" state. (B) After activation of the Hh ligand, Hh proteins are released from the signaling cell. Hh then subsequently binds ("On" state) to PtcH, removing the inhibition and further activating SMO. SMO then regulates the downstream transduction molecules of Gli proteins (Gli I, Gli2, and Gli3). Gli proteins are subsequently transferred to the nuclei and they exert their transduction functions.

The cause of CRC is still not clear; however, there are multiple factors involved in the formation and development of CRC, including age, dietary habits, genetic alteration (mutational activation of oncogenes and inhibition of several tumor suppressor genes), epithelial-to-mesenchymal transformation (EMT) and its reversal in cancer invasion and mucosal healing, and angiogenesis in tumor growth and metastasis. ${ }^{34-36}$ Extensive studies have been conducted to explore the molecular mechanisms underlying the tumorigenesis of CRC. Various signaling mutations have been confirmed to contribute to CRC development, including KRAS, MYB, and BRAF (Table 1). ${ }^{17,19-21}$ Moreover, Gut 
Table I Mutations that are correlated in colorectal carcinogenesis

\begin{tabular}{|c|c|c|c|c|}
\hline Gene & $\begin{array}{l}\text { Chromosomal } \\
\text { location }\end{array}$ & Type of mutation & Prevalence (\%) & Function of gene product \\
\hline KRAS & $12 p \mid 2$ & Point mutation (codons 12,13 of exon 2 ) & 40 & Cell proliferation and survival \\
\hline PIK3CA & $3 q 26$ & $\begin{array}{l}\text { Point mutations (E545K on exon } 9 \\
\text { HI047R on exon } 20 \text { ) }\end{array}$ & $15-30$ & Cell proliferation and survival \\
\hline CDK8 & $13 q \mid 2$ & Gene amplification & $10-15$ & $\beta$-Catenin activation \\
\hline EGFR & $7 p 12$ & Gene amplification & $5-15$ & Cell proliferation and survival \\
\hline BRAF & $7 q 34$ & $\begin{array}{l}\text { Point mutations activating kinase activity } \\
\text { (most commonly } \mathrm{V} 600 \mathrm{E} \text { ) }\end{array}$ & $5-10$ & Cell proliferation and survival \\
\hline CMYC & $8 q 24$ & Gene amplification & $5-10$ & Cell proliferation and survival \\
\hline CCNEI & $19 q 12$ & Gene amplification & 5 & Cell proliferation and survival \\
\hline NRAS & $|p| 3$ & Point mutation & $<5$ & Cell proliferation and survival \\
\hline CTNNBI & $3 p 22$ & $\begin{array}{l}\text { Stabilizing point mutations and in-frame } \\
\text { deletions near } N \text { terminus }\end{array}$ & $<5$ & $\begin{array}{l}\text { Regulation of Wnt pathway target genes } \\
\text { that promote tumor growth and invasion }\end{array}$ \\
\hline ERBB2 (HER2) & $|7 q 2|$ & Gene amplification & $<5$ & Cell proliferation and survival \\
\hline MYB & $6 q 22-q 23$ & Gene amplification & $<5$ & Stimulates growth of intestinal stem cells \\
\hline
\end{tabular}

flora disorder and inflammatory diseases also contribute to CRC generation. ${ }^{22}$

It has now been well illustrated that $\mathrm{CRC}$ is derived from the intestinal epithelium and arises as a consequence of the progressive accumulation of genetic and epigenetic changes that drive the transformation and progression of normal colorectal epithelial cells to carcinoma. ${ }^{37,38}$ In CRC, cells follow an ordered sequence of events called "adenomacarcinoma sequence", which starts with the transformation of normal colorectal epithelium to an adenomatous intermediate and then to the adenocarcinoma phase. ${ }^{39}$ The pathogenesis of CRC generally ends with the formation of invasive carcinomas that usually metastasize to the liver. ${ }^{32}$

Growing evidence supports the concept that epithelial cancers, including $\mathrm{CRC}$, are diseases driven by the pluripotent, self-renewing cancer stem cells (CSCs). ${ }^{40} \mathrm{CSCs}$ represent the apex in the hierarchical model of tumor genesis, heterogeneity, and metastasis. They possess the capacity of unlimited self-renewal through symmetric cell division, the ability to give rise to progeny cells through asymmetric division, and an innate resistance to cytotoxic therapeutics. ${ }^{41} \mathrm{Wnt}$, Notch, Hh, and/or TGF- $\beta$ signaling pathways are involved in proliferation and maintenance of CSCs, and dysregulation of these pathways might cause the development of CRC. ${ }^{42-45}$ Despite the advancements in diagnosis and targeted and combined treatment, the advanced and metastatic stage of CRC still remains untreatable. Further development in targeting treatment and prevention is one of the major challenges. ${ }^{36}$ Prevention of CRC includes various measures including lifestyle modification, identification and early intervention of individuals at risk, and treatment of pre-neoplastic lesions. ${ }^{37}$ Some drugs such as aspirin are also reported to be effective in CRC prevention; however, the clinical usage of such drugs was limited due to some side effects. ${ }^{38}$

The prognosis of CRC varies according to factors such as the stage at diagnosis, treatment quality, and individual health status. Identification of new prognostic biomarkers could be a useful tool in predicting disease development and making personalized treatment plans. ${ }^{48} \mathrm{With}$ the help of TCGA data and other approaches, now studies have located numbers of molecules that might be used as biomarkers. ${ }^{114-117}$ Besides the expected biomarkers such as SMAD family member 4, APC, tumor protein p53, phosphatidylinositol4,5-bisphosphate 3-kinase catalytic subunit alpha, and KRAS proto-oncogene (KRAS) mutations, frequent mutations in $\mathrm{AT}$-rich interaction domain $1 \mathrm{~A}$, sex determining region Y-box 9, and FAM123B/WTX were detected in CRC, indicating that these could be used as specific biomarkers for CRC. ${ }^{116}$

The most important prognostic indicator is stage at diagnosis. The 5-year relative survival of patients diagnosed with $\mathrm{CRC}$ is $90 \%$ for patients with disease localized to the original sites, $69 \%$ for patients with regional spread, and less than $12 \%$ for patients with metastasis. ${ }^{37} \mathrm{CRCs}$ are classified according to local invasion depth ( $\mathrm{T}$ stage), lymph node involvement ( $\mathrm{N}$ stage), and distant metastases ( $\mathrm{M}$ stage). ${ }^{38}$ These TNM stages provide most valuable prognostic information and basic treatment guidance. Nevertheless, the outcome and response of individual patient to therapy are variable. ${ }^{39}$

\section{Hh signaling pathway in CRC}

Aberrant activation of the Hh signaling pathway is associated with tumorigenesis in various tissues. ${ }^{46} \mathrm{Up}$ until 
now, however, the role of the Hh signaling pathway in the formation and progression of CRC still remains controversial among the studies and researches. ${ }^{44,47-49}$ Its exact involvement in the formation, growth, and metastasis of CRC remains enigmatic. Some studies showed no direct correlation between abnormal Hh pathway activation and CRC cells. ${ }^{25-27}$ Chatel et al examined the Hh signaling pathway component expression in $7 \mathrm{CRC}$ cell lines and found that aberrant activation of the Hh signaling pathway is not involved in CRC cell lines. ${ }^{26}$ Alinger et al also concluded that Hh signaling is involved in differentiation and renewing of the colonic lining epithelium instead of cancer formation, growth, or proliferation. ${ }^{27}$ However, such studies either lacked large quantity samplings or failed to acquire a coherent conclusion, and thus are unconvincing.

Through decades of studying and researching, the mainstream idea concerning the role of the Hh signaling pathway in CRC can be concluded by the following aspects: 1) different types of CRCs and CRC cells exhibit different expressions of components in the $\mathrm{Hh}$ signaling pathway; 2) the $\mathrm{Hh}$ pathway may function in gene mutation, EMT and metastasis, angiogenesis to alter the cell malignancy in CRC; 3) the roles of Hh signaling pathway differ at each stage during the adenoma-to-adenocarcinoma development of CRC; 4) Shh would promote the development of CRC, whilst Ihh would inhibit $\mathrm{CRC}$ formation; and 5) the downstream component of the Hh signaling pathway, Smo, may have the most important role in Hh regulation of CRC..$^{21,48-50}$

\section{Hh signaling pathway participates in tumorigenesis of CRC}

Despite some studies that fail to conclude a positive correlation of $\mathrm{Hh}$ signaling pathway and the development of $\mathrm{CRC}$, the major body of concerning studies has provided a positive correlation between them. Most studies specify that the Hh signaling pathway participates in oncogenesis of CRC. Hh signaling regulates colonic enterocyte differentiation, and the Hh mRNA and protein are highly expressed in CRC cell lines. ${ }^{51-53}$ An active Hh-Gli pathway is also found to be displayed in epithelial tumor cells in most human CRC cell lines. ${ }^{54}$ Most scholars agreed that a high Hh-SmoGli activity is acquired in CRC for tumor cell survival and metastasis, and that the Hh signaling is activated by both canonical signaling (via Smo) and non-canonical activation (via the RAS/RAF pathway) in colon cancers. ${ }^{32,55,56}$

Transcriptional regulation of the Hh signaling response is mediated by Gli genes (Gli1, Gli2) downstream of Smo, which are also activated by oncogenic signaling pathways.
Switching off Hh signaling at the level of Gli induces extensive cell death, in contrast to targeting Smo upstream of Gli. ${ }^{57}$ Sénicourt et al further confirmed the participation of the Hh signaling pathway by finding that cilia are present in CRC cells with high expression of Smo and Gli. ${ }^{58}$

\section{Different types of CRCs and CRC cells exhibit different expression of components in Hh signaling pathway}

From previous studies we can find that the activation of the Hh pathway is highly incongruent in different CRC types and CRC cell lines, implying that the function of the Hh pathway may be variable according to different CRC cancer types.

For instance, $\mathrm{Li}$ et $\mathrm{al}^{1}$ discovered that Smo expression was not statistically correlated with CRC-specific or overall survival, while in $\mathrm{CpG}$ island methylator phenotype-high tumors, CRC-specific survival was distinctively associated with higher Smo expression. ${ }^{67}$ The study by Stefanius et al revealed no correlation between $\mathrm{Hh}$ and colorectal serrated adenocarcinomas. ${ }^{59} \mathrm{Hu}$ et al also reported that $\mathrm{Hh}$ signals were more frequently expressed in the microsatellite instable group, compared with the microsatellite stable group in CRC. ${ }^{60}$ All those studies described that the activation of the Hh signaling pathway is restricted in several types of $\mathrm{CRC}$, but not in other types of cancer.

\section{The Hh signaling pathway may function in gene mutation, EMT and metastasis, cell differentiation, and angiogenesis to alter the malignancy in CRC}

The Hh signaling pathway was shown to be multi-functional in the formation of CRC. The involvement of the Hh pathway in gene mutation, EMT and metastasis, apoptosis, and angiogenesis was discovered. Oku et al found that Hh signal is correlated with dedifferentiation at the initial metastasis sites of CRC. ${ }^{62}$

Activation of the Hh signaling pathway is also reported to be associated with Gli1-induced lymphangiogenesis and tumor cell regeneration in $\mathrm{CRC}$, which is correlated to the metastatic ability and drug resistance in chemotherapy of CRCs. ${ }^{46,63,64}$

\section{The participation of Hh signaling pathway differs at each stage in the adenoma- adenocarcinoma process}

The development of CRC is now commonly proved by most studies to go through the adenoma-adenocarcinoma process. 
Accordingly, the role and importance of Hh signaling pathway vary during each stage of the process. ${ }^{49,65}$ Zhang et al found that Smo and Gli1 expressions were enhanced gradually from the normal colon to colonic adenoma and then to the CRC, implying that the role of the Hh pathway may be increasingly enhanced during the process of CRC tumorigenesis. ${ }^{44} \mathrm{Xu}$ et al noticed that expression of Shh, Ptc, and Gli1 mRNA was gradually increased along the Peutz-Jeghers polyposis (PJP)-adenoma-adenocarcinoma sequence. ${ }^{66} \mathrm{Peng}$ et al also suggested that aberrant methylation of the Ptc1 promoter would inhibit the expression of Ptc1, which may be an early, initiating event of colon carcinogenesis. ${ }^{67}$

\section{Shh would promote the development of CRC, whilst lhh would inhibit CRC formation}

The Hh signaling pathway conducted by two homologs of Shh and Ihh is of central relevance in cancer genesis. ${ }^{68,69}$ Most studies have confirmed the stimulatory function of Shh and inhibitory function of Ihh in CRC formation. ${ }^{70}$

Shh pathway has been reported to have stimulatory function in the process of angiogenesis, cell proliferation, inhibition of tumor suppression genes, and metastasis. ${ }^{71-74}$ Many studies have proved that Shh is expressed in CSCs and CRC cell and tumor masses and showed a positive correlation between Shh overexpression and CRC tumorigenesis. ${ }^{47,75-80}$ $\mathrm{Xu}$ et al examined the expression of Shh, Ptc, and Gli1 in 20 normal tissues and 75 colorectal lesions (25 PJPs, 25 adenomas, and 25 adenocarcinomas) and found that overexpression of Shh may be responsible for the elevated expression of Gli1 in colorectal neoplasms. ${ }^{66}$ The results of the clinical trial conducted by Yoshikawa et al indicated that Shh can contribute to the process of adenomaadenocarcinoma aggravation. ${ }^{81}$

In many studies, downregulation of Ihh has been observed as an early event in the formation of CRC..$^{82,83}$ van den Brink et al found that loss of Ihh expression precedes the development of dysplasia in colon carcinogenesis. ${ }^{51}$ Gerling et al concluded that Ihh is mainly expressed in stromal tissue in colon. Increased Ihh expression will inhibit the formation of $\mathrm{CRC}$, and stromal Hh can be used as a tumor suppressor. ${ }^{84}$ The mechanism of the inhibitory function of Ihh in CRC tumorigenesis is still not quite lucid. van den Brink et al specifically pointed out that Ihh antagonizes Wnt signaling in colonic epithelial cell differentiation. ${ }^{51}$ Further studies also confirmed that the activation of Wnt might contribute to the downregulation or loss of Ihh expression in colorectal tumors. ${ }^{85}$ Some also raised a hypothesis that upregulation of Ihh expression may work against CRC by inducing differentiation of tumor cells and abrogating the Shh signaling that drives $\mathrm{CRC}$ growth. ${ }^{86}$

\section{The downstream components of $\mathrm{Hh}$ signaling pathway, such as Smo and Gli, may have the most important role in Hh regulation of $C R C$}

Plenty of studies have pointed out that the downstream components of the Hh signaling pathway are the key to the tumorigenesis of CRC, and the cooperation of Smo and Gli has the most important role in Hh regulation of CRC. . $^{30,66,87-89}$ Further studies also confirmed that the Hh signaling pathway components Smo and Gli exhibit coordinated expression in colon cancer cell lines..$^{90}$ These results give rise to the idea that the downstream components of the Hh pathway may play even more important roles in the tumorigenesis of CRC than the upstream components such as Hh and Ptc, which provides the theoretical basis for the potential of targeting inhibition of Hh signaling at the level of the Gli genes, downstream of Smo, in the treatment of human colon carcinoma cells. $^{91}$

\section{Hh signaling pathway may network with other mechanisms and signaling transductions to cooperate in the tumorigenesis of CRC}

The development of CRC is a complicated process involving various mechanisms and factors, and requires a complex of network among different signal transductions. The Hh signaling pathway is also involved in the signaling transductions to orchestration in the tumorigenesis of CRC..$^{71,92}$

Early events in the progression of $90 \%$ of sporadic CRCs depend on constitutive activation of Wnt signaling. ${ }^{93}$ The role of Wnt signaling is well established in colorectal carcinogenesis. ${ }^{94,95}$ Activation of both $\mathrm{Wnt} / \beta$-catenin and $\mathrm{Hh} / \mathrm{Gli1}$ signaling pathways results in the overexpression of cancer-related genes such as cyclin D and Myc (c-Myc), which are involved in cancer development of several malignancies. ${ }^{96}$ Both the Wnt and Hh signaling pathways are essential for the normal development of gastrointestinal tissues, and most studies are prone to the thought that they are related to tumorigenesis in gastrointestinal tumors, including CRC. ${ }^{15,97-100}$ A study found out that Hh signaling pathway is seldom activated in CRC due to inhibitory regulation by the canonical Wnt signaling pathway, suggesting that Wnt may play a regulating role in the Hh pathway in CRC. ${ }^{14}$ 
Moreover, several studies proved that Ihh functions as an antagonist of Wnt signaling during colorectal tumorigenesis, and the Hh signaling pathway was recently shown to antagonize the constitutive activity of Wnt pathway that drives proliferation and metastasis of CRC cells, thus establishing an initial antagonizing counterpart between Ihh and Wnt pathways. ${ }^{12,15,33,37}$ In human CRC, enhanced Gli1 represses Wnt-TCF targets and is involved in the metastasis of CRC. ${ }^{101-105} \mathrm{Hh}$ signaling in intestinal epithelium represses canonical Wnt signaling to restrict expression of Wnt target genes in stem or progenitor cells. ${ }^{48}$ The current research supports the idea that downregulation of Ihh-Ptc-Gli1 signaling is essential to allow unrestrained Wnt signaling and progression to adenocarcinoma. ${ }^{18}$ Some studies also reported that Smo can increase Wnt signaling. ${ }^{87,106}$

\section{Hh inhibitors in the treatment of CRC}

Although the relationship between the Hh pathway and CRC remains inconclusive, the application of Hh inhibitors has been practiced in cellular, animal, and clinical experiments. ${ }^{107}$ For instance, Meng et al reported that overexpression of $\mathrm{SuFu}$, an inhibitory regulator of the Hh signaling pathway in SW480 (APC ${ }^{\mathrm{mut}}$ ) colon cancer cells, could inhibit cancer cell growth and tumor formation in nude mice. ${ }^{108}$

The most widely used Hh inhibitor in clinical practice is cyclopamine. Various trials have been conducted to testify the effectiveness of cyclopamine in treating CRC. Many showed promising results, and some found that cyclopamine can significantly reduce apoptosis and decrease proliferation in CRC cells. ${ }^{31,32}$ Qualtrough et al found that cyclopamine treatment can induce the expression of E-Cadherin in both benign and malignant colorectal tumor cell lines and can reduce the invasion ability in SW480 cells. ${ }^{109}$ Besides cyclopamine, cabozantinib is also reported to effectively reduce EMT potential on another CRC cell line HCT-116's spheres and tumor size and angiogenesis, and suppressed the expression of vascular endothelial growth factor in tumor tissues. ${ }^{10,111}$ Moreover, one trial on the oral investigational Hh signaling pathway inhibitor TAK-441 in patients revealed an antitumor activity of the Hh inhibitor TAK-441 in advanced CRC. ${ }^{113}$

Nevertheless, some studies reported that Hh inhibitor cyclopamine did not affect viability of these colon cancer cell lines. ${ }^{15}$ Some even showed opposite conclusion and pointed out that Hh antagonists may promote colonic carcinogenesis, induce hypergastrinemia, and lead to increased numbers of Paneth cells with unknown effects on mucosal immunity and that Hh agonists may instead be effective in preventing or treating colon cancer. ${ }^{18}$
In conclusion, Hh inhibitor is promising in treating gastrointestinal carcinoma, but more details about the mechanism of Hh inhibitors in the process of the Hh signaling pathway are needed. Inhibition of different sites in the pathway could be useful in different types of CRC, and thus requires deeper development in Hh inhibitors, more various types of $\mathrm{Hh}$ inhibitors and more extensive research into the treatment effect of Hh inhibitors in CRC. ${ }^{112}$ Nevertheless, most of the currently available research on the anti-oncogenic effects of $\mathrm{Hh}$ inhibitors in CRCs are limited to in vitro and in vivo levels. Further explorations of the effectiveness in human are required to confirm the clinical effect and acquire more knowledge on Hh functions of tumorigenesis.

\section{Discussion}

Based on all the researches and findings, we concluded that the Hh signaling pathway may play a crucial role in the tumorigenesis of CRCs. ${ }^{4-35,40,42,47-49,53-113}$ Although its exact role remains controversial, the expression of the $\mathrm{Hh}$ pathway in CRC tissues is much higher than in normal colon tissues, especially Shh and its signaling pathway components. Removals of certain parts in Shh pathway could lead to failure of cell development, proliferation, metastasis, and tumor sustenance in CRC, according to multiple studies. ${ }^{17-24}$

Shh is now considered to likely promote formation and metastasis of CRCs. ${ }^{24,35}$ It is believed that overexpression of Shh and its downstream components is highly correlated with the formation and metastasis of CRCs, whereas it's detailed mechanism remains unclear. Some studies revealed that it is a paracrine factor and works with the inhibition of anti-oncogenes, like p53. ${ }^{41}$ More studies showed that it is also linked to other important signaling pathways, like Wnt/ beta-catenin; together they perform the function of sustaining and proliferation of CRC cells and lead to the final formation of CRCs. ${ }^{11,14,37}$ Ihh is another important ligand in Hh family. Research shows that it is mainly expressed in stromal tissues in the colon, and it has the suppressive effect of CRC. ${ }^{92}$ More studies are required for further exploration of the $\mathrm{Hh}$ pathway and its function in $\mathrm{CRCs} .{ }^{66}$

Hh inhibitor is considered as one of the therapeutic methods in treating cancers. Its application in treating CRC has been reported and received promising results, implying that the development of signaling pathway targeting treatment in CRC is a promising path for antitumor treatment. ${ }^{27,113}$ More details about the mechanism of Hh inhibitors in the Hh pathway are needed. Inhibition of different sites in the pathway could be useful in different types of CRC. ${ }^{89}$ 
Thus, deeper development of Hh inhibitors, more various types of $\mathrm{Hh}$ inhibitors and more extensive research on the treatment effect of $\mathrm{Hh}$ inhibitors in CRC is needed.

\section{Acknowledgments}

This work was supported by the National Natural Science Foundation of China (NSFC) (No 81600401; 81572413), Wuhan Scientific and Technological Application Foundation Project (2015060101010044), and Health and Family Plan Committee of China Research Fund of Public welfare in Health Industry (201402015).

\section{Author contributions}

All authors contributed toward data analysis, drafting and critically revising the paper and agree to be accountable for all aspects of the work.

\section{Disclosure}

The authors report no conflicts of interest in this work.

\section{References}

1. Li T, Liao X, Lochhead P, et al. SMO expression in colorectal cancer: associations with clinical, pathological, and molecular features. Ann Surg Oncol. 2014;21(13):4164-4173.

2. Taipale J, Beachy PA. The Hedgehog and Wnt signalling pathways in cancer. Nature. 2001;411(6835):349-354.

3. Ingham PW, McMahon AP. Hedgehog signaling in animal development: paradigms and principles. Genes Dev. 2001;15(23):3059-3087.

4. Mehlen P, Tauszig-Delamasure S. Dependence receptors and colorectal cancer. Gut. 2014;63(11):1821-1829.

5. Konstantinou D, Bertaux-Skeirik N, Zavros Y. Hedgehog signaling in the stomach. Curr Opin Pharmacol. 2016;31:76-82.

6. Papadopoulos V, Tsapakidis K, Riobo Del Galdo NA, et al. The prognostic significance of the Hedgehog signaling pathway in colorectal cancer. Clin Colorectal Cancer. 2016;15(2):116-127.

7. Seow HF, Yip WK, Fifis T. Advances in targeted and immunobased therapies for colorectal cancer in the genomic era. Onco Targets Ther. 2016;9:1899-1920.

8. Haveri H, Westerholm-Ormio M, Lindfors K, et al. Transcription factors GATA-4 and GATA-6 in normal and neoplastic human gastrointestinal mucosa. BMC Gastroenterol. 2008;8:9.

9. Katoh Y, Katoh M. Comparative genomics on Sonic hedgehog orthologs. Oncol Rep. 2005;14(4):1087-1090.

10. Lin J, Chen Y, Cai Q, et al. Scutellaria barbata D Don inhibits colorectal cancer growth via suppression of multiple signaling pathways. Integr Cancer Ther. 2014;13(3):240-248.

11. Parfitt JR, Driman DK. Survivin and hedgehog protein expression in serrated colorectal polyps: an immunohistochemical study. Hum Pathol. 2007;38(5):710-717.

12. Watt FM. Unexpected Hedgehog-Wnt interactions in epithelial differentiation. Trends Mol Med. 2004;10(12):577-580.

13. Wong H, Alicke B, West KA, et al. Pharmacokinetic-pharmacodynamic analysis of vismodegib in preclinical models of mutational and liganddependent Hedgehog pathway activation. Clin Cancer Res. 2011; 17(14):4682-4692.

14. Katoh Y, Katoh M. Hedgehog signaling pathway and gastrointestinal stem cell signaling network (review). Int J Mol Med. 2006;18(6): 1019-1023.
15. van den Brink GR, Hardwick JC. Hedgehog Wnteraction in colorectal cancer. Gut. 2006;55(7):912-914.

16. Ruiz i Altaba A. Hedgehog signaling and the Gli code in stem cells, cancer, and metastases. Sci Signal. 2011;4(200):pt9.

17. Agyeman A, Jha BK, Mazumdar T, Houghton JA. Mode and specificity of binding of the small molecule GANT61 to GLI determines inhibition of GLI-DNA binding. Oncotarget. 2014;5(12):4492-4503.

18. Lees CW, Satsangi J. Hedgehog, Paneth cells, and colon cancer: a cautionary note for the use of systemic agonists/antagonists. Gastroenterology. 2006;131(5):1657-1658.

19. Chowdhury S, Pradhan RN, Sarkar RR. Structural and logical analysis of a comprehensive hedgehog signaling pathway to identify alternative drug targets for glioma, colon and pancreatic cancer. PLoS One. 2013;8(7):e69132.

20. Ciucci A, De Stefano I, Vellone VG, et al. Expression of the gliomaassociated oncogene homolog 1 (gli1) in advanced serous ovarian cancer is associated with unfavorable overall survival. PLoS One. 2013; 8(3):e60145.

21. Chung JH, Larsen AR, Chen E, Bunz F. A PTCH1 homolog transcriptionally activated by $\mathrm{p} 53$ suppresses Hedgehog signaling. J Biol Chem. 2014;289(47):33020-33031.

22. Delloye-Bourgeois C, Gibert B, Rama N, et al. Sonic Hedgehog promotes tumor cell survival by inhibiting CDON pro-apoptotic activity. PLoS Biol. 2013;11(8):e1001623.

23. Zhao L, Mao Y, Zhou J, Zhao Y, Cao Y, Chen X. Multifunctional DDX3: dual roles in various cancer development and its related signaling pathways. Am J Cancer Res. 2016;6(2):387-402.

24. Chung JH, Bunz F. A loss-of-function mutation in PTCH1 suggests a role for autocrine hedgehog signaling in colorectal tumorigenesis. Oncotarget. 2013;4(12):2208-2211.

25. Guleng B, Tateishi K, Ohta M, et al. Smoothened gene mutations found in digestive cancer have no aberrant Hedgehog signaling activity. J Gastroenterol. 2006;41(12):1238-1239.

26. Chatel G, Ganeff C, Boussif N, et al. Hedgehog signaling pathway is inactive in colorectal cancer cell lines. Int J Cancer. 2007;121(12): 2622-2627.

27. Alinger B, Kiesslich T, Datz C, et al. Hedgehog signaling is involved in differentiation of normal colonic tissue rather than in tumor proliferation. Virchows Arch. 2009;454(4):369-379.

28. IJssennagger N, Rijnierse A, de Wit N, et al. Dietary haem stimulates epithelial cell turnover by downregulating feedback inhibitors of proliferation in murine colon. Gut. 2012;61(7):1041-1049.

29. Song J, Zhang J, Wang J, Wang J, Guo X, Dong W. $\beta 1$ integrin mediates colorectal cancer cell proliferation and migration through regulation of the Hedgehog pathway. Tumour Biol. 2015;36(3):2013-2021.

30. Gulino A, Ferretti E, De Smaele E. Hedgehog signalling in colon cancer and stem cells. EMBO Mol Med. 2009;1(6-7):300-302.

31. Wu JY, Xu XF, Xu L, et al. Cyclopamine blocked the growth of colorectal cancer SW116 cells by modulating some target genes of Gli1 in vitro. Hepatogastroenterology. 2011;58(110-111):1511-1518.

32. Varnat F, Duquet A, Malerba M, et al. Human colon cancer epithelial cells harbour active HEDGEHOG-GLI signalling that is essential for tumour growth, recurrence, metastasis and stem cell survival and expansion. EMBO Mol Med. 2009;1(6-7):338-351.

33. Joyce T, Oikonomou E, Kosmidou V, et al. A molecular signature for oncogenic BRAF in human colon cancer cells is revealed by microarray analysis. Curr Cancer Drug Targets. 2012;12(7):873-898.

34. Sipos F, Galamb O. Epithelial-to-mesenchymal and mesenchymalto-epithelial transitions in the colon. World J Gastroenterol. 2012; 18(7):601-608.

35. Zhu J, Liu C, Liu F, et al. Knockdown of PFTAIRE protein kinase 1 (PFTK1) inhibits proliferation, invasion, and EMT in colon cancer cells. Oncol Res. 2016;24(3):137-144.

36. Subramaniam D, Ramalingam S, Houchen CW, Anant S. Cancer stem cells: a novel paradigm for cancer prevention and treatment. Mini Rev Med Chem. 2010;10(5):359-371. 
37. Kolligs FT. Diagnostics and epidemiology of colorectal cancer. Visc Med. 2016;32(3):158-164.

38. Hirose H, Ishii H, Mimori K, et al. The significance of PITX2 overexpression in human colorectal cancer. Ann Surg Oncol. 2011; 18(10):3005-3012.

39. Centelles JJ. General aspects of colorectal cancer. ISRN Oncol. 2012. Epub 2012 Nov 14.

40. Ramasamy TS, Ayob AZ, Myint HH, Thiagarajah S, Amini F. Targeting colorectal cancer stem cells using curcumin and curcumin analogues: insights into the mechanism of the therapeutic efficacy. Cancer Cell Int. 2015;15:96.

41. Curtin JC, Lorenzi MV. Drug discovery approaches to target Wnt signaling in cancer stem cells. Oncotarget. 2010;1(7):552-566.

42. Roy S, Majumdar AP. Signaling in colon cancer stem cells. $J$ Mol Signal. 2012;7(1):11.

43. Ramírez A, Boulaiz H, Morata-Tarifa C, et al. HER2-signaling pathway, JNK and ERKs kinases, and cancer stem-like cells are targets of Bozepinib small compound. Oncotarget. 2014;5(11): 3590-3606.

44. Zhang X, Zhang SS, Wei GJ, Deng ZM, Hu Y. Dysregulation of hedgehog signaling pathway related components in the evolution of colonic carcinogenesis. Int J Clin Exp Med. 2015;8(11):21379-21385.

45. So JY, Suh N. Targeting cancer stem cells in solid tumors by vitamin D. J Steroid Biochem Mol Biol. 2015;148:79-85.

46. Hong KD, Lee Y, Kim BH, Lee SI, Moon HY. Expression of GLI1 correlates with expression of lymphangiogenesis proteins, vascular endothelial growth factor $\mathrm{C}$ and vascular endothelial growth factor receptor 3, in colorectal cancer. Am Surg. 2013;79(2):198-204.

47. Douard R, Moutereau S, Pernet P, et al. Sonic Hedgehog-dependent proliferation in a series of patients with colorectal cancer. Surgery. 2006; 139(5):665-670.

48. Katoh Y, Katoh M. WNT antagonist, SFRP1, is Hedgehog signaling target. Int J Mol Med. 2006;17(1):171-175.

49. Bian YH, Huang SH, Yang L, Ma XL, Xie JW, Zhang HW. Sonic hedgehog-Glil pathway in colorectal adenocarcinomas. World $J$ Gastroenterol. 2007;13(11):1659-1665.

50. Wang H, Ke F, Zheng J. Hedgehog-glioma-associated oncogene homolog-1 signaling in colon cancer cells and its role in the celecoxibmediated anti-cancer effect. Oncol Lett. 2014;8(5):2203-2208.

51. van den Brink GR, Bleuming SA, Hardwick JC, et al. Indian Hedgehog is an antagonist of Wnt signaling in colonic epithelial cell differentiation. Nat Genet. 2004;36(3):277-282.

52. Shi T, Mazumdar T, Devecchio J, et al. cDNA microarray gene expression profiling of hedgehog signaling pathway inhibition in human colon cancer cells. PLoS One. 2010;5(10):e13054.

53. Taniguchi H, Yamamoto H, Akutsu N, et al. Transcriptional silencing of hedgehog-interacting protein by $\mathrm{CpG}$ hypermethylation and chromatic structure in human gastrointestinal cancer. J Pathol. 2007;213(2) $131-139$

54. You S, Zhou J, Chen S, et al. PTCH1, a receptor of Hedgehog signaling pathway, is correlated with metastatic potential of colorectal cancer. Ups J Med Sci. 2010;115(3):169-175.

55. Cai X, Yu K, Zhang L, et al. Synergistic inhibition of colon carcinoma cell growth by Hedgehog-Gli1 inhibitor arsenic trioxide and phosphoinositide 3-kinase inhibitor LY294002. Onco Targets Ther. 2015;8: 877-883.

56. Mazumdar T, DeVecchio J, Agyeman A, Shi T, Houghton JA. The GLI genes as the molecular switch in disrupting Hedgehog signaling in colon cancer. Oncotarget. 2011;2(8):638-645.

57. Agyeman A, Mazumdar T, Houghton JA. Regulation of DNA damage following termination of Hedgehog $(\mathrm{HH})$ survival signaling at the level of the GLI genes in human colon cancer. Oncotarget. 2012;3(8): 854-868.

58. Sénicourt B, Boudjadi S, Carrier JC, Beaulieu JF. Neoexpression of a functional primary cilium in colorectal cancer cells. Heliyon. 2016;2(5): e00109.
59. Stefanius K, Kantola T, Tuomisto A, et al. Downregulation of the hedgehog receptor PTCH1 in colorectal serrated adenocarcinomas is not caused by PTCH1 mutations. Virchows Arch. 2011;458(2):213-219.

60. Hu X, Lai D, Chen W, et al. Differential expression profiles of the Hedgehog signaling pathway between microsatellite-stable and microsatellite-unstable colorectal cancers. Mol Med Rep. 2011;4(5): 873-877.

61. Yoshimoto AN, Bernardazzi C, Carneiro AJ, et al. Hedgehog pathway signaling regulates human colon carcinoma HT-29 epithelial cell line apoptosis and cytokine secretion. PLoS One. 2012;7(9):e45332.

62. Oku Y, Shimoji T, Takifuji K, et al. Identification of the molecular mechanisms for dedifferentiation at the invasion front of colorectal cancer by a gene expression analysis. Clin Cancer Res. 2008;14(22): $7215-7222$.

63. Ma J, Tian L, Cheng J, et al. Sonic hedgehog signaling pathway supports cancer cell growth during cancer radiotherapy. PLoS One. 2013; 8(6): 65032

64. Liu Y, Du F, Zhao Q, Jin J, Ma X, Li H. Acquisition of 5-fluorouracil resistance induces epithelial-mesenchymal transitions through the Hedgehog signaling pathway in HCT-8 colon cancer cells. Oncol Lett. 2015;9(6):2675-2679.

65. Heijink DM, Fehrmann RS, de Vries EG, et al. A bioinformatical and functional approach to identify novel strategies for chemoprevention of colorectal cancer. Oncogene. 2011;30(17):2026-2036.

66. Xu X, Su J, Li R, Wang Y, Zeng D, Wu B. Aberrant expression of Sonic hedgehog signaling in Peutz-Jeghers syndrome. Hum Pathol. 2016;50:153-161.

67. Peng L, Hu J, LiS, et al. Aberrant methylation of the PTCH1 gene promoter region in aberrant crypt foci. Int J Cancer. 2013;132(2):E18-E25.

68. Klaus C, Jeon MK, Kaemmerer E, Gassler N. Intestinal acyl-CoA synthetase 5: activation of long chain fatty acids and behind. World $J$ Gastroenterol. 2013;19(42):7369-7373.

69. McMahon AP, Ingham PW, Tabin CJ. Developmental roles and clinical significance of hedgehog signaling. Curr Top Dev Biol. 2003; $53: 1-114$.

70. Fu X, Deng H, Zhao L, Li J, Zhou Y, Zhang Y. Distinct expression patterns of hedgehog ligands between cultured and primary colorectal cancers are associated with aberrant methylation of their promoters. Mol Cell Biochem. 2010;337(1-2):185-192.

71. Wei L, Lin J, Xu W, et al. Scutellaria barbata D. Don inhibits tumor angiogenesis via suppression of Hedgehog pathway in a mouse model of colorectal cancer. Int J Mol Sci. 2012;13(8):9419-9430.

72. Lin J, Chen Y, Wei L, Hong Z, Sferra TJ, Peng J. Ursolic acid inhibits colorectal cancer angiogenesis through suppression of multiple signaling pathways. Int J Oncol. 2013;43(5):1666-1674.

73. Lin J, Wei L, Shen A, et al. Hedyotis diffusa Willd extract suppresses Sonic hedgehog signaling leading to the inhibition of colorectal cancer angiogenesis. Int J Oncol. 2013;42(2):651-656.

74. Li L, Lin J, Sun G, et al. Oleanolic acid inhibits colorectal cancer angiogenesis in vivo and in vitro via suppression of STAT3 and Hedgehog pathways. Mol Med Rep. 2016;13(6):5276-5282.

75. Wang H, Li YY, Wu YY, Nie YQ. Expression and clinical significance of hedgehog signaling pathway related components in colorectal cancer. Asian Pac J Cancer Prev. 2012;13(5):2319-2324.

76. Kangwan N, Kim YJ, Han YM, Jeong M, Park JM, Hahm KB. Concerted actions of ameliorated colitis, aberrant crypt foci inhibition and 15-hydroxyprostaglandin dehydrogenase induction by sonic hedgehog inhibitor led to prevention of colitis-associated cancer. Int J Cancer. 2016;138(6):1482-1493.

77. Al-Bahrani R, Nagamori S, Leng R, Petryk A, Sergi C. Differential expression of sonic hedgehog protein in human hepatocellular carcinoma and intrahepatic cholangiocarcinoma. Pathol Oncol Res. 2015; 21(4):901-908.

78. Oniscu A, James RM, Morris RG, Bader S, Malcomson RD, Harrison DJ. Expression of Sonic hedgehog pathway genes is altered in colonic neoplasia. J Pathol. 2004;203(4):909-917. 
79. Wang TP, Hsu SH, Feng HC, Huang RF. Folate deprivation enhances invasiveness of human colon cancer cells mediated by activation of sonic hedgehog signaling through promoter hypomethylation and cross action with transcription nuclear factor-kappa B pathway. Carcinogenesis. 2012;33(6):1158-1168.

80. Monzo M, Moreno I, Artells R, et al. Sonic hedgehog mRNA expression by real-time quantitative PCR in normal and tumor tissues from colorectal cancer patients. Cancer Lett. 2006;233(1):117-123.

81. Yoshikawa K, Shimada M, Miyamoto H, et al. Sonic hedgehog relates to colorectal carcinogenesis. J Gastroenterol. 2009;44(11):1113-1117.

82. Fu X, Yang X, Li J, Tian X, Cai J, Zhang Y. Opposite expression patterns of Sonic hedgehog and Indian hedgehog are associated with aberrant methylation status of their promoters in colorectal cancers. Pathology. 2010;42(6):553-559.

83. Büller NV, Rosekrans SL, Metcalfe C, et al. Stromal Indian hedgehog signaling is required for intestinal adenoma formation in mice. Gastroenterology. 2015;148(1):170-180.e6.

84. Gerling M, Büller NV, Kirn LM, et al. Stromal Hedgehog signalling is downregulated in colon cancer and its restoration restrains tumour growth. Nat Commun. 2016;7:12321.

85. Fu X, Shi L, Zhang W, et al. Expression of Indian hedgehog is negatively correlated with APC gene mutation in colorectal tumors. Int J Clin Exp Med. 2014;7(8):2150-2155.

86. Fu X, Yang X, Zhao L. Indian hedgehog, a neglected member of hedgehog pathway, may offer a novel avenue for colorectal cancer therapy. Cancer Biother Radiopharm. 2009;24(6):733-735.

87. Arimura S, Matsunaga A, Kitamura T, Aoki K, Aoki M, Taketo MM. Reduced level of smoothened suppresses intestinal tumorigenesis by down-regulation of Wnt signaling. Gastroenterology. 2009;137(2): 629-638.

88. Ding YL, Wang QS, Zhao WM, Xiang L. Expression of smoothened protein in colon cancer and its prognostic value for postoperative liver metastasis. Asian Pac J Cancer Prev. 2012;13(8):4001-4005.

89. Iwasaki H, Nakano K, Shinkai K, et al. Hedgehog Gli3 activator signal augments tumorigenicity of colorectal cancer via upregulation of adherence-related genes. Cancer Sci. 2013;104(3):328-336.

90. Zhu Y, James RM, Peter A, et al. Functional smoothened is required for expression of GLI3 in colorectal carcinoma cells. Cancer Lett. 2004; 207(2):205-214.

91. Mazumdar T, Devecchio J, Agyeman A, Shi T, Houghton JA. Blocking Hedgehog survival signaling at the level of the GLI genes induces DNA damage and extensive cell death in human colon carcinoma cells. Cancer Res. 2011;71(17):5904-5914.

92. Kang HN, Oh SC, Kim JS, Yoo YA. Abrogation of Gli3 expression suppresses the growth of colon cancer cells via activation of p53. Exp Cell Res. 2012;318(5):539-549.

93. Wu WK, Wang XJ, Cheng AS, et al. Dysregulation and crosstalk of cellular signaling pathways in colon carcinogenesis. Crit Rev Oncol Hematol. 2013;86(3):251-277.

94. Dellinger TH, Planutis K, Tewari KS, Holcombe RF. Role of canonical Wnt signaling in endometrial carcinogenesis. Expert Rev Anticancer Ther. 2012;12(1):51-62.

95. Saif MW, Chu E. Biology of colorectal cancer. Cancer J. 2010;16(3): 196-201.

96. Song L, Liu J, Jin X, Li Z, Zhao M, Liu W. p, p'-Dichlorodiphenyld ichloroethylene induces colorectal adenocarcinoma cell proliferation through oxidative stress. PLoS One. 2014;9(11):e112700.

97. Bertrand FE, Angus CW, Partis WJ, Sigounas G. Developmental pathways in colon cancer: crosstalk between WNT, BMP, Hedgehog and Notch. Cell Cycle. 2012;11(23):4344-4351.

98. Tateishi K, Ohta M, Kanai F, et al. Dysregulated expression of stem cell factor Bmil in precancerous lesions of the gastrointestinal tract. Clin Cancer Res. 2006;12(23):6960-6966.
99. Fanale D, Barraco N, Listì A, Bazan V, Russo A. Non-coding RNAs functioning in colorectal cancer stem cells. Adv Exp Med Biol. 2016; 937:93-108.

100. Hasson RM, Briggs A, Carothers AM, et al. Estrogen receptor $\alpha$ or $\beta$ loss in the colon of Min/+ mice promotes crypt expansion and impairs TGF $\beta$ and HNF3 $\beta$ signaling. Carcinogenesis. 2014;35(1):96-102.

101. Mimeault M, Batra SK. Potential applications of curcumin and its novel synthetic analogs and nanotechnology-based formulations in cancer prevention and therapy. Chin Med. 2011;6:31.

102. Akiyoshi T, Nakamura M, Koga K, et al. Gli1, downregulated in colorectal cancers, inhibits proliferation of colon cancer cells involving Wnt signalling activation. Gut. 2006;55(7):991-999.

103. Noubissi FK, Goswami S, Sanek NA, et al. Wnt signaling stimulates transcriptional outcome of the Hedgehog pathway by stabilizing GLI1 mRNA. Cancer Res. 2009;69(22):8572-8578.

104. Aberger F, Ruiz I, Altaba A. Context-dependent signal integration by the GLI code: the oncogenic load, pathways, modifiers and implications for cancer therapy. Semin Cell Dev Biol. 2014;33:93-104.

105. Mehmood K, Akhtar D, Mackedenski S, Wang C, Lee CH. Inhibition of GLI1 expression by targeting the CRD-BP-GLI1 mRNA interaction using a specific oligonucleotide. Mol Pharmacol. 2016;89(6): 606-617.

106. Song L, Li ZY, Liu WP, Zhao MR. Crosstalk between Wnt/ $\beta$-catenin and Hedgehog/Gli signaling pathways in colon cancer and implications for therapy. Cancer Biol Ther. 2015;16(1):1-7.

107. Lettre G, Jackson AU, Gieger C, et al. Identification of ten loci associated with height highlights new biological pathways in human growth. Nat Genet. 2008;40(5):584-591.

108. Meng X, Poon R, Zhang X, et al. Suppressor of fused negatively regulates beta-catenin signaling. J Biol Chem. 2001;276(43): 40113-40119.

109. Qualtrough D, Rees P, Speight B, Williams AC, Paraskeva C. The hedgehog inhibitor cyclopamine reduces $\beta$-catenin-Tcf transcriptional activity, induces E-cadherin expression, and reduces invasion in colorectal cancer cells. Cancers (Basel). 2015;7(3):1885-1899.

110. Sun Y, Sun L, An Y, Shen X. Cabozantinib, a novel c-Met inhibitor, inhibits colorectal cancer development in a xenograft model. Med Sci Monit. 2015;21:2316-2321.

111. Batsaikhan BE, Yoshikawa K, Kurita N, et al. Cyclopamine decreased the expression of Sonic Hedgehog and its downstream genes in colon cancer stem cells. Anticancer Res. 2014;34(11):6339-6344.

112. Wiedmann MW. Molecular targeted therapy of gastrointestinal cancer. Curr Cancer Drug Targets. 2011;11(6):670-680.

113. Goldman J, Eckhardt SG, Borad MJ, et al. Phase I dose-escalation trial of the oral investigational Hedgehog signaling pathway inhibitor TAK441 in patients with advanced solid tumors. Clin Cancer Res. 2015; 21(5):1002-1009.

114. Abetov D, Mustapova Z, Saliev T, Bulanin D. Biomarkers and signaling pathways of colorectal cancer stem cells. Tumour Biol. 2015; 36(3):1339-1353.

115. Mahasneh A, Al-Shaheri F, Jamal E. Molecular biomarkers for an early diagnosis, effective treatment and prognosis of colorectal cancer: current updates. Exp Mol Pathol. 2017;102(3):475-483.

116. Cancer Genome Atlas Network. Comprehensive molecular characterization of human colon and rectal cancer. Nature. 2012;487(7407): 330-337.

117. Zhang F, Ren C, Lau KK, et al. A network medicine approach to build a comprehensive atlas for the prognosis of human cancer. Brief Bioinform. 2016;17(6):1044-1059. 
OncoTargets and Therapy

\section{Publish your work in this journal}

OncoTargets and Therapy is an international, peer-reviewed, open access journal focusing on the pathological basis of all cancers, potential targets for therapy and treatment protocols employed to improve the management of cancer patients. The journal also focuses on the impact of management programs and new therapeutic agents and protocols on

patient perspectives such as quality of life, adherence and satisfaction. The manuscript management system is completely online and includes a very quick and fair peer-review system, which is all easy to use. Visit http://www.dovepress.com/testimonials.php to read real quotes from published authors.

Submit your manuscript here: http://www.dovepress.com/oncotargets-and-therapy-journal 